\title{
Examination of Inspection Method for Outer-side Defect on Ferromagnetic Steel Tube by Velocity Effect Using Insertion-type Static Magnetic Field Sensor
}

\author{
Makoto Tohara, ${ }^{1 *}$ Masafumi Kuromizu, ${ }^{2}$ and Yuji Gotoh ${ }^{3 * *}$ \\ ${ }^{1}$ Department of Technical Development, TOA Non-Destructive Inspection Co., Ltd., \\ 8-46 Shinsone, Kitakyushu 800-0211, Japan \\ ${ }^{2}$ Department of Mechatronics Engineering, Oita University, 700 Dannoharu, Oita 870-1192, Japan \\ ${ }^{3}$ Department of Innovative Engineering, Faculty of Science and Technology, Oita University, \\ 700 Dannoharu, Oita 870-1192, Japan
}

(Received June 15, 2021; accepted July 24, 2021)

Keywords: ferromagnetic steel tube, outer-side defect, static magnetic field, eddy current, 3D nonlinear FEM

When an insertion-type magnetic sensor using only a static magnetic field is moved in a steel tube at a high speed, an eddy current is generated in the steel. When there is a defect in the steel tube, it is possible to detect the defect from the change in the distribution of the eddy current in the tube. Generally, ferromagnetic heat transfer tubes in power plants or oil plants are inspected for defects by moving an internally inserted electromagnetic sensor at a high speed of about $1 \mathrm{~m} / \mathrm{s}$. In this study, an inspection method is proposed in which an insertion-type electromagnetic sensor using only a static magnetic field is moved at a high speed in a steel tube to detect an outer-side defect in the tube. The eddy current density and alternating flux density inside the steel tube generated by constant velocity movement of the static magnetic field are determined by 3D nonlinear finite element method (FEM) analysis of the eddy current by a step-by-step method. In addition, a verification experiment is also conducted.

\section{Introduction}

An insertion-type sensor is required for the high-speed inspection of the inside of heat transfer steel tubes of multitubular heat exchangers in chemical or oil plants. Eddy current testing (ECT) using a minute alternating magnetic field is applied to inspect non-magnetic tubes in chemical or oil plants at a high speed without contact. ${ }^{(1)}$ Since a high excitation frequency is used in this inspection method, the inside of the steel tube is usually inspected with the sensor moving at a high speed of about $1 \mathrm{~m} / \mathrm{s}$ inside the steel tube. However, when normal ECT is applied to ferromagnetic steel tubes, since the skin effect is strong in this method, a defect on the outer side of the steel tube is not detected.

On the other hand, when a static magnetic field is applied to a ferromagnetic steel tube from the inside of the steel tube, the magnetic flux penetrates to the outside of the tube. Therefore, an inspection method using both a static magnetic field and ECT is also being studied. ${ }^{(2,3)}$ However,

*Corresponding author: e-mail: v18f1001@oita-u.ac.jp

**Corresponding author: e-mail: goto-yuuji@oita-u.ac.jp

https://doi.org/10.18494/SAM.2021.3463 
when an electromagnetic sensor using only a static magnetic field is moved in a steel tube at a high speed, an outer-side defect on the steel tube is detected since an eddy current is generated inside the steel tube. Inspection methods using the velocity effect with a static magnetic field have already been proposed. ${ }^{(4-7)}$ However, in most previous studies, these inspection methods using the velocity effect were applied to non-magnetic materials such as aluminum.

In this study, an electromagnetic inspection method using the velocity effect with a static magnetic field in which a sensor is inserted into a steel tube is investigated to detect an outerside defect in a ferromagnetic steel tube. The flux density and eddy current density in this inspection method are examined by the 3D nonlinear finite element method (FEM) taking account of the initial $B-H$ curve in the steel tube and the velocity effect of the insertion-type static magnetic sensor. ${ }^{(8)}$ In addition, the usefulness of this inspection method is experimentally verified.

\section{Sensor Model and Analysis Method}

\subsection{Electromagnetic sensor model and conditions}

Figure 1 shows the proposed electromagnetic sensor using only a static magnetic field and an inspection steel tube (STB340SC). This sensor is composed of two static excitation coils in series, a pair of differential detection coils, and a magnetic yoke of Permendur, an alloy of iron and cobalt. A direct current of $1 \mathrm{~A}$ is passed through the two excitation coils in series so that the positive $z$-direction of the sensor is the S-pole and the negative $z$-direction is the $\mathrm{N}$-pole. The two

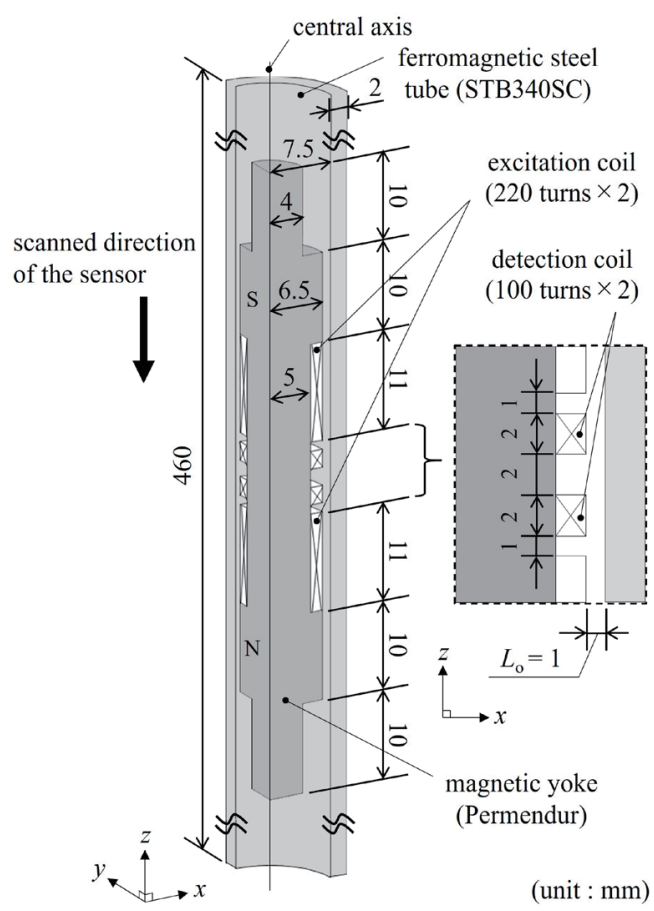

Fig. 1. Electromagnetic sensor model (1/4 of domain). 
excitation coils and the differential detection coil are 220 turns $\times 2$ and 100 turns $\times 2$, respectively. The outer diameter, length, and thickness of this inspection steel tube are $\phi 19 \mathrm{~mm}$, $460 \mathrm{~mm}$, and $2 \mathrm{~mm}$, respectively. The proposed sensor is inserted in the steel tube at a speed of $1 \mathrm{~m} / \mathrm{s}$ in the negative $z$-direction. The distance (lift-off: $L_{o}$ ) between the sensor and the steel tube is $1 \mathrm{~mm}$.

\subsection{Nonlinear analysis considering moving speed}

In this inspection method, the flux density and eddy current density are calculated by the 3D electromagnetic FEM taking account of the initial magnetic curves and the conductivities of the steel tube and the magnetic yoke. The 3D FEM using first-order hexahedral edge elements is applied.

The basic equation of the electromagnetic field analysis in consideration of the eddy current using the $\boldsymbol{A}-\phi$ method is given by

$$
\begin{gathered}
\operatorname{rot}(v \operatorname{rot} \boldsymbol{A})=\boldsymbol{J}_{0}-\sigma\left(\frac{\partial \boldsymbol{A}}{\partial t}+\operatorname{grad} \phi\right) \\
\operatorname{div}\left\{-\sigma\left(\frac{\partial \boldsymbol{A}}{\partial t}+\operatorname{grad} \phi\right)\right\}=0,
\end{gathered}
$$

where $\boldsymbol{A}$ is the magnetic vector potential, $\phi$ is the scalar potential, $v$ is the reluctivity, $\boldsymbol{J}_{0}$ is the current density, and $\sigma$ is the conductivity. To briefly explain the calculation considering the velocity effect of the sensor, we examine the case of movement with a constant velocity in the $z$-direction, as shown in Fig. 2, with a 1D model without considering the $\operatorname{grad} \phi$ term in Eqs. (1)

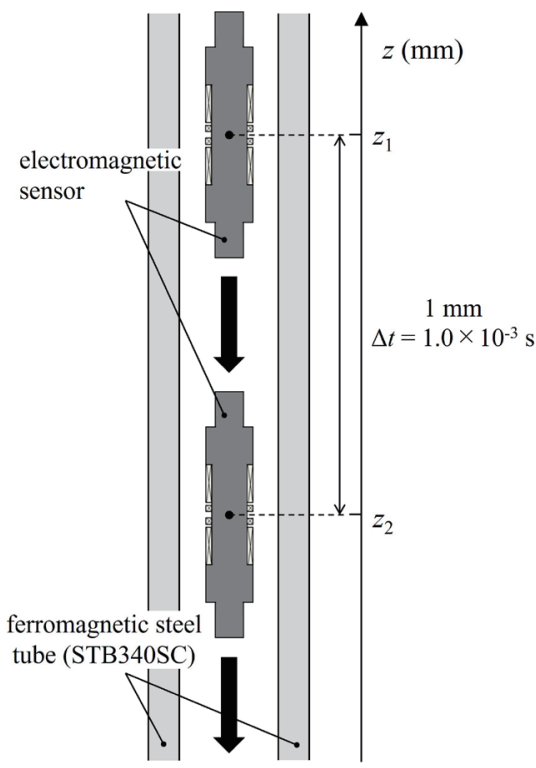

Fig. 2. Movement of sensor in $z$-direction. 
and (2). In transient analysis, where the sensor with the static magnetic field is moved inside the steel tube over time, the time derivative $\partial A / \partial t$ for the moving coordinate system can be discretized by the backward difference method that is superior to the other methods as follows: ${ }^{(9,10)}$

$$
\frac{\partial A\left(z_{2}\right)^{t+\Delta t}}{\partial t} \cong \frac{A^{*}\left(z_{2}\right)^{t+\Delta t}-A\left(z_{1}\right)^{t}}{\Delta t}
$$

where $\Delta t$ is the time interval. $z_{1}$ indicates an arbitrary position of the sensor, and $z_{2}$ indicates the position of the sensor after $\Delta t$ from the position of $z_{1}$. For example, $A\left(z_{2}\right)^{t+\Delta t}$ represents the vector potential of an arbitrary position after movement at the instant $t+\Delta t$. The superscript (*) indicates the unknown variable. Since the speed of the sensor moving in the steel tube is $1 \mathrm{~m} / \mathrm{s}$ and the moving pitch in the FEM analysis is $1 \mathrm{~mm} / \mathrm{step}$ in the negative $z$-direction, the time interval $\Delta t$ in the step-by-step method is selected as $1.0 \times 10^{-3} \mathrm{~s}$. In 3D analysis, the same discretization as above is carried out for all directions. The Newton-Raphson $(N-R)$ method is used for the nonlinear iterative calculation of the magnetic characteristic. The $N-R$ iterations are carried out using the initial magnetization curve shown in Fig. 3. The incomplete Cholesky Conjugate Gradient (ICCG) method is used to converge the results of nonlinear calculations of the magnetic characteristics. The conditions of calculation and measurement are shown in Table 1.

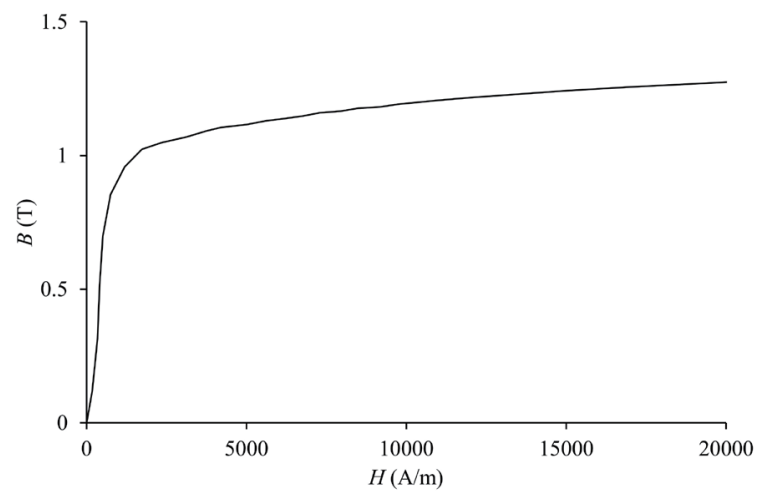

Fig. 3. Initial magnetization curve of ferromagnetic steel tube (STB340SC).

Table 1

Conditions of calculation and measurement.

\begin{tabular}{lc}
\hline Excitation coil & 220 turns $\times 2$, DC: $1 \mathrm{~A}$ \\
\hline Differential search coil & 100 turns $\times 2$ \\
\hline Scanning speed of the sensor & $1 \mathrm{~m} / \mathrm{s}$ \\
\hline Lift-off & $1 \mathrm{~mm}$ \\
\hline Dimensions of specimen & $\begin{array}{c}\text { STB340SC steel tube, diameter: } 19 \mathrm{~mm}, \\
\text { thickness: } 2 \mathrm{~mm}, \text { length: } 460 \mathrm{~mm}\end{array}$ \\
\hline Conductivity & $\begin{array}{l}\text { STB340SC: } 6.66 \times 10^{6} \mathrm{~S} / \mathrm{m}, \\
\text { Permendur: } 3.33 \times 10^{6} \mathrm{~S} / \mathrm{m}\end{array}$ \\
\hline Nodes and elements & 107597,100452 \\
\hline Convergence criterion & $N-R$ method: $1.0 \times 10^{-6} \mathrm{~T}$, \\
\end{tabular}




\section{Magnetic Flux and Eddy Current Distribution in Steel Tube During Constant Velocity Movement}

Figure 4 shows the distribution of the eddy current density in one layer in the $y$-direction inside the steel tube without defect when the sensor is moved in the negative $z$-direction in the tube at $1 \mathrm{~m} / \mathrm{s}$. This figure indicates that the directions of the eddy currents generated on the upper and lower sides of the steel tube are opposite. Since the lower part of the sensor in the moving direction is the N-pole, the eddy current is generated inside the steel tube near the lower part of the sensor in the direction that cancels the N-pole magnetic field. On the other hand, since the upper part of this sensor is the S-pole, an eddy current is generated inside the steel tube near the sensor in the direction that cancels the S-pole magnetic field. In addition, owing to the skin effect, the eddy currents are concentrated on the inner surface layer within the thickness of the steel tube.

Figure 5 illustrates the display domain of the magnetic flux density in the steel tube shown in the analysis results below. Figure 6 shows the distribution of the flux density inside domain 1 of the steel tube without a defect in Fig. 5 when the sensor is moved in the negative $z$-direction in the tube at $1 \mathrm{~m} / \mathrm{s}$. Figure 6(a) shows the distribution of the flux density inside the steel tube without a defect generated by the velocity effect. This figure indicates that the flux density on the outer surface of the steel tube is reduced. Also, the flux density is distributed in the positive $z$-direction since the eddy current is generated inside the steel tube in the direction that cancels the $N$-pole magnetic field. Figure 6(b) shows only the static flux density component inside the

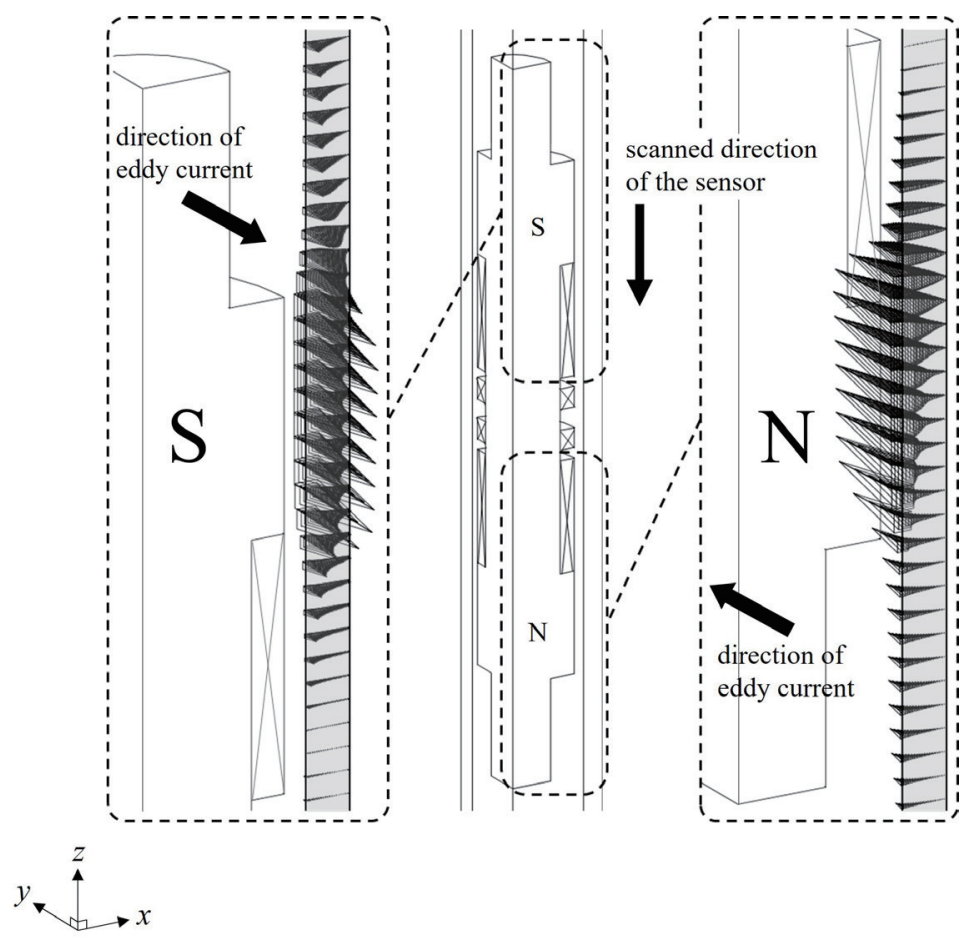

Fig. 4. Distribution of eddy current density in the steel tube at a moving speed of $1 \mathrm{~m} / \mathrm{s}$. 


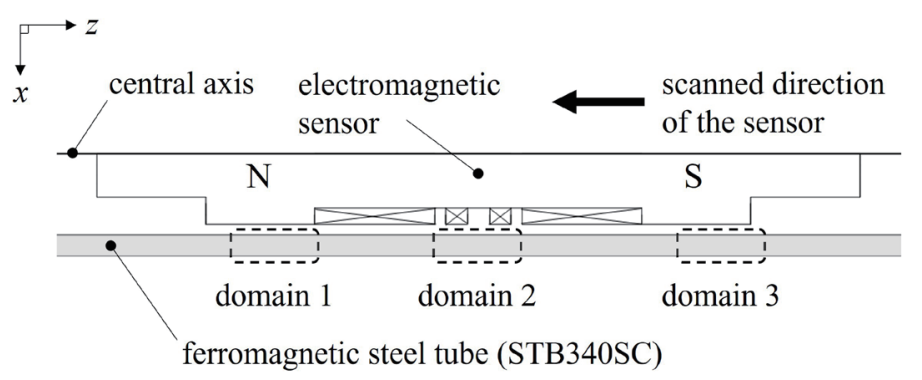

Fig. 5. Display domain of magnetic flux density in the steel tube.

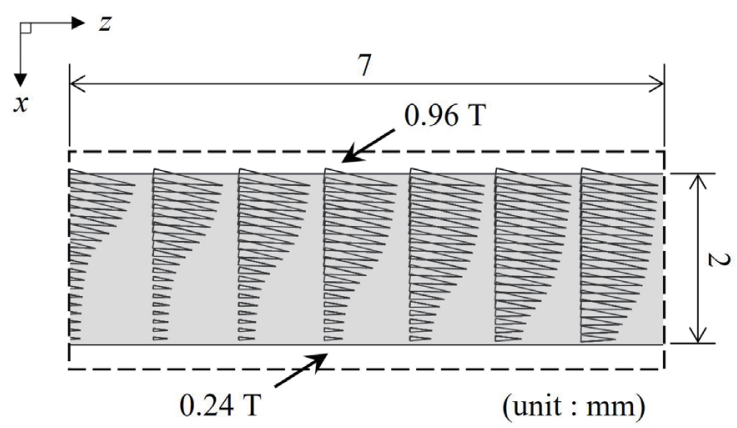

(a)

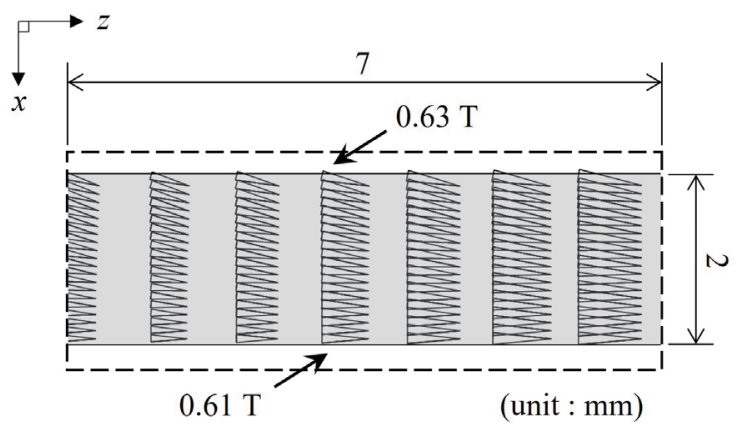

(b)

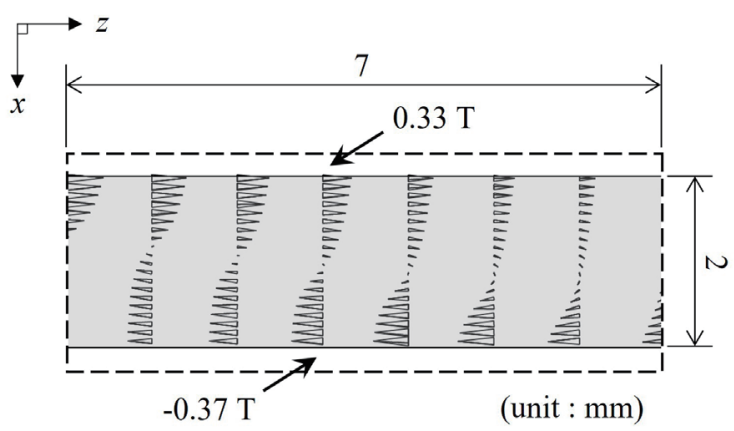

(c)

Fig. 6. Distribution of flux density in domain 1 of ferromagnetic steel tube. (a) Flux density generated by velocity effect. (b) Static flux density component. (c) AC flux density component due to eddy current.

steel tube generated by the velocity effect as shown in Fig. 6(a). This figure indicates that the static flux density component is uniformly distributed inside the steel tube. Figure 6(c) shows the AC flux density component obtained by subtracting the static flux density component in Fig. 6(b) from the flux density distribution in the steel tube generated by the velocity effect as shown in Fig. 6(a). This figure indicates the AC flux density component induced by the velocity effect is concentrated on the inner surface of the steel tube by the skin effect. In addition, the distribution of the flux density component on the outer surface of the steel tube is the static flux density component reduced by the skin effect. 
Figure 7 shows the distribution of the flux density inside domain 2 of the steel tube without a defect when the sensor is moved in the negative $z$-direction in the tube at $1 \mathrm{~m} / \mathrm{s}$. Figure 7 (a) shows the distribution of flux density inside the steel tube generated by the velocity effect. This figure indicates that the magnetic flux density is uniformly distributed inside the steel tube. Figure 7(b) shows only the static flux density component inside the steel tube generated by the velocity effect as shown in Fig. 7(a). This figure indicates that the static flux density is uniformly distributed inside the steel tube as shown in Fig. 7(a). Figure 7(c) shows the AC flux density component obtained by subtracting the static flux density component in Fig. 7(b) from the flux density distribution in the steel tube generated by the velocity effect as shown in Fig. 7(a). This figure indicates that the $\mathrm{AC}$ magnetic flux density inside the steel tube near the central region of the sensor is very small, since eddy current is not almost generated in the steel tube near the central region of the sensor field as shown in Fig. 4. Therefore, Fig. 7 shows that the distribution of static flux density in the steel tube near the central region of the sensor is dominant.

Figure 8 shows the distribution of the flux density inside domain 3 of the steel tube without a defect when the sensor is moved in the negative $z$-direction in the tube at $1 \mathrm{~m} / \mathrm{s}$. Figure 8 (a) shows the distribution of flux density inside the steel tube generated by the velocity effect. This figure indicates that the flux density on the inner surface of the steel tube is reduced. In addition, it indicates that the flux density on the inner surface of the steel tube near the upper side of the sensor is offset because the eddy current is generated in the opposite direction to that when the $\mathrm{N}$-pole of the lower side of the sensor passed as shown in Fig. 4.

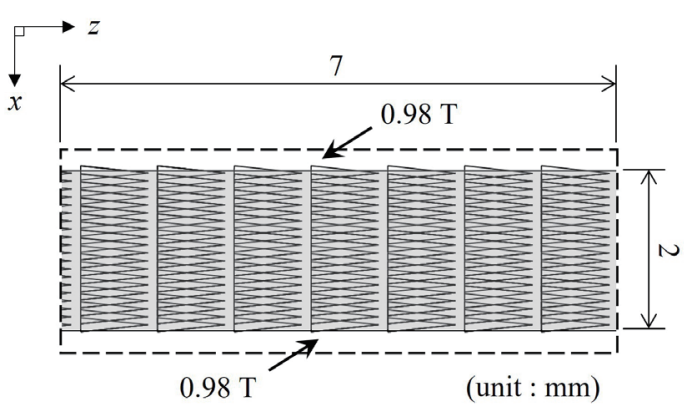

(a)

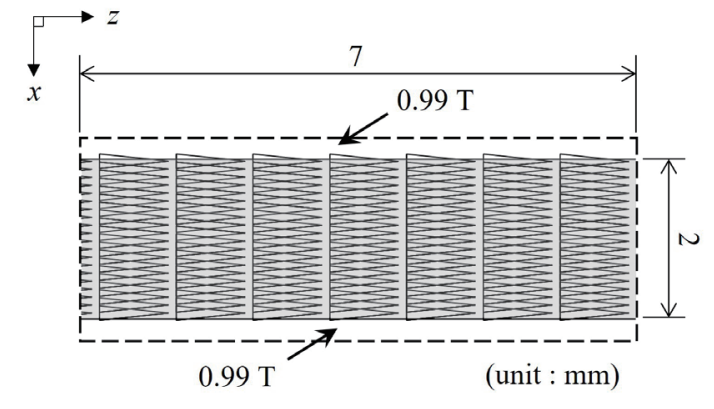

(b)

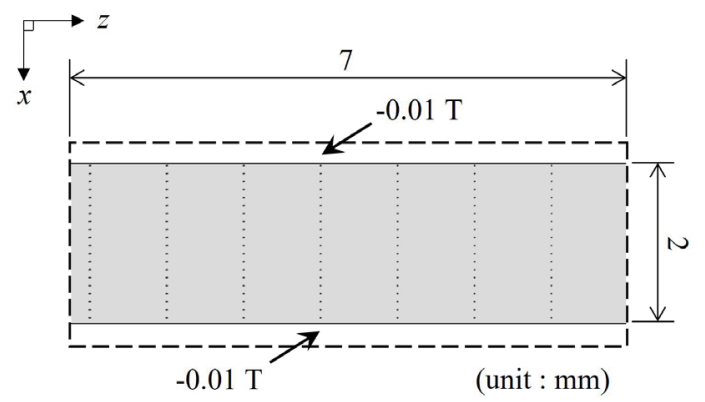

(c)

Fig. 7. Distribution of flux density in domain 2 of ferromagnetic steel tube. (a) Flux density generated by velocity effect. (b) Static flux density component. (c) AC flux density component due to eddy current. 


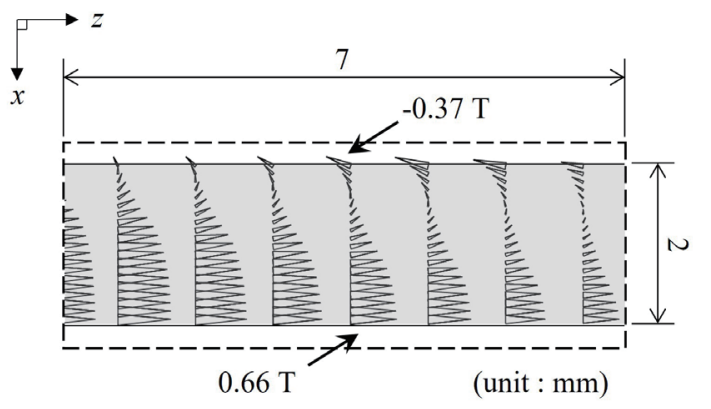

(a)

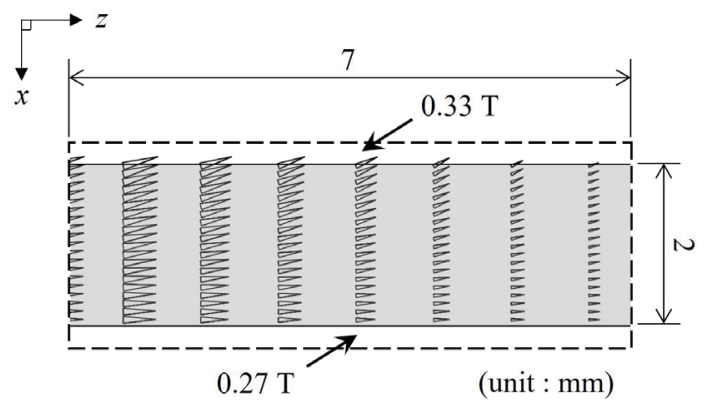

(b)

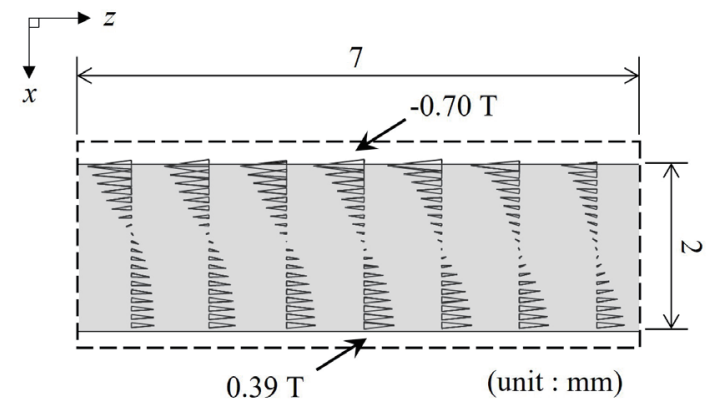

(c)

Fig. 8. Distribution of flux density in domain 3 of ferromagnetic steel tube. (a) Flux density generated by velocity effect. (b) Static flux density component. (c) AC flux density component due to eddy current.

Figure 8(b) shows only the static flux density inside the steel tube generated by the velocity effect as shown in Fig. 8(a). This figure indicates that the static flux density is uniformly distributed inside the steel tube. Figure 8(c) shows the AC flux density component obtained by subtracting the static flux density component of Fig. 8(b) from the flux density distribution in the steel tube generated by the velocity effect as shown in Fig. 8(a). This figure indicates that the AC flux density component generated by the velocity effect is concentrated on the inner surface of the steel tube by the skin effect. In addition, the distribution of the flux density component on the outer surface of the steel tube is the static flux density component increased by the skin effect.

\section{Verification of Outer-side Defect Inspection by Calculation and Experiment}

\subsection{Conditions of verification experiment}

The verification model and the conditions of the calculation and experiment are basically the same as those shown in Fig. 1 and Table 1. The current used for excitation is DC 1 A. However, the center of the outer-side defect is set as $z=0 \mathrm{~mm}$, and the proposed electromagnetic sensor is moved in the $z$-direction from 30 to $-30 \mathrm{~mm}$ at a speed of $1 \mathrm{~m} / \mathrm{s}$ as shown in Fig. 9. In this experiment, the recording interval (sampling interval) of the voltmeter is set to $0.1 \mathrm{~ms}$, the sensor 


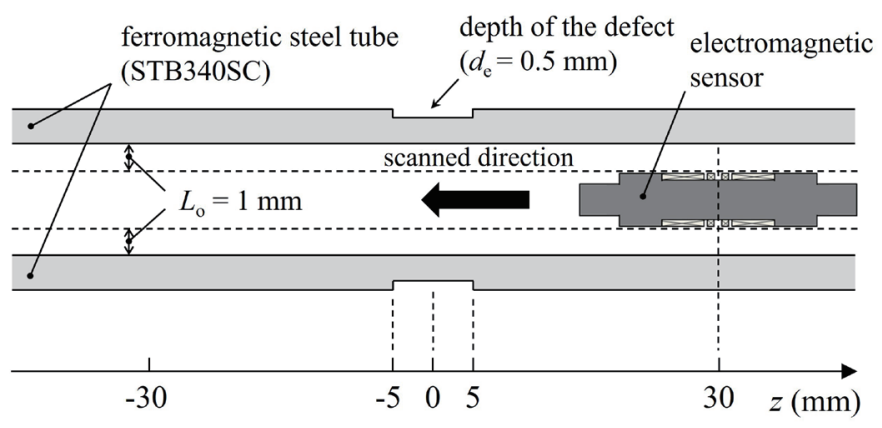

Fig. 9. Model for detecting defect using proposed electromagnetic sensor.

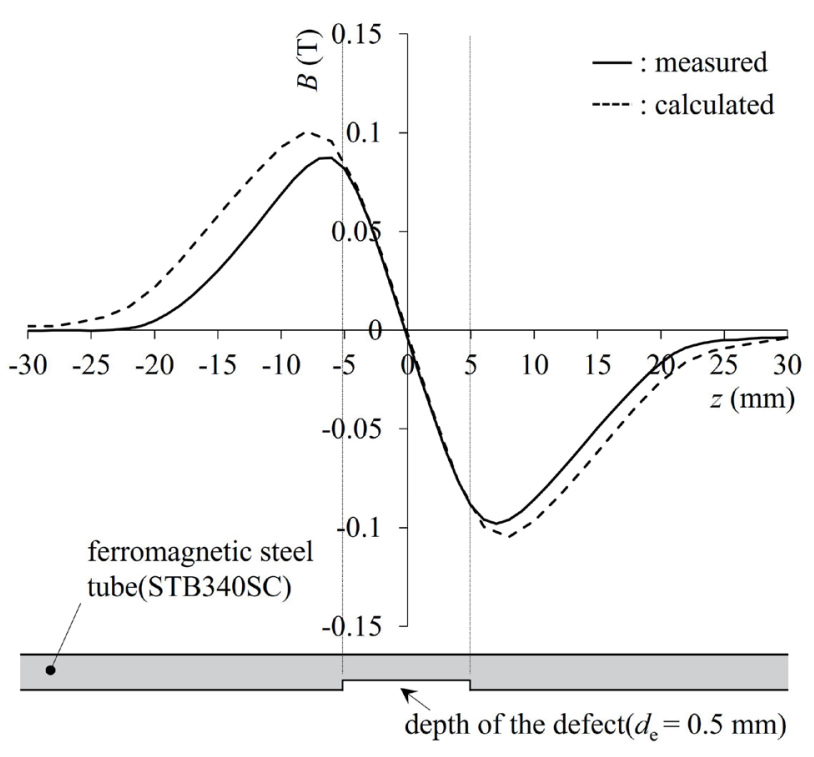

Fig. 10. Inspection results of the outer-side defect on the steel tube.

is fixed, and the steel tube is moved at $1 \mathrm{~m} / \mathrm{s}$ using a motorized scanning machine. This sensor measures the differential output voltage in two detection coils at each moving position while maintaining the lift-off $L_{o}$ at $1 \mathrm{~mm}$. The outer-side defect on the steel tube is a circumferential defect with a width of $1 \mathrm{~mm}$ in the $z$-direction and a depth of $0.5 \mathrm{~mm}$ in the $x$-direction.

\subsection{Inspection results}

Figure 10 shows the inspection signal of the differential flux density detected by the two detection coils for the outer-side defect on the steel tube. The figure also shows the FEM analysis results. This figure indicates that the outer-side defect is detected since the two peaks appear near both edges of the defect. In addition, the calculated differential flux density in the two detection coils is almost in agreement with the measured values.

Figure 11 shows the distribution of the eddy current density within the steel tube with and without the defect when the center of the electromagnetic sensor is located at $z=12 \mathrm{~mm}$ for the 


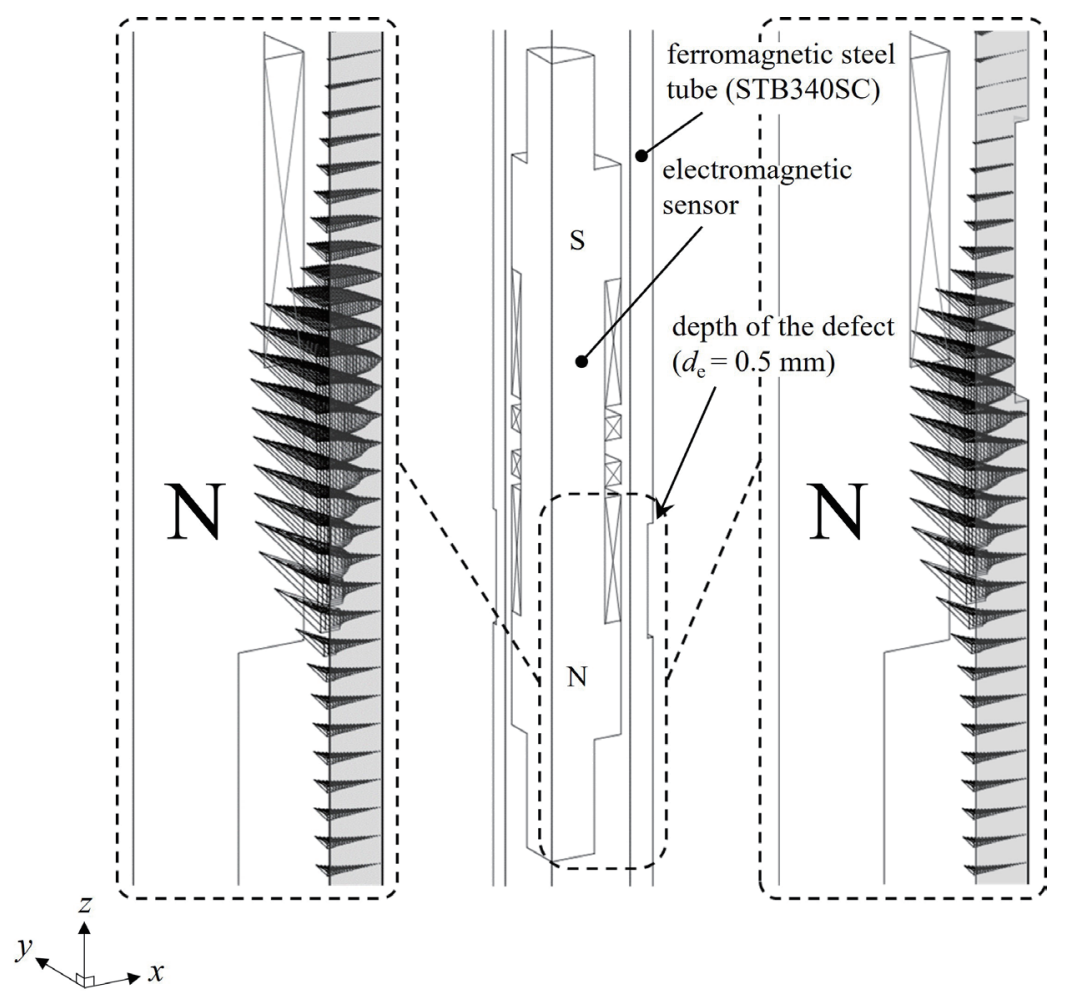

Fig. 11. Distribution of eddy current density in the steel tube with and without the defect $(z=12 \mathrm{~mm})$. (a) Without the defect $\left(\left|J_{e}\right|_{\max }=9.8 \times 10^{5} \mathrm{~A} / \mathrm{m}^{2}\right)$. (b) With the defect $\left(\left|J_{e}\right|_{\max }=9.6 \times 10^{5} \mathrm{~A} / \mathrm{m}^{2}\right)$.

detection signal shown Fig. 10. The figure indicates that when there is an outer-side defect on the steel tube, the eddy current generated by the velocity effect is decreased. Therefore, since the difference between the eddy currents inside the steel tube near the N-pole side and the S-pole side in the sensor is increased, a signal is generated in the differential detection coil.

\section{Conclusions}

The results obtained in this study are summarized as follows:

1. The flux density and eddy current density inside a steel tube generated by the proposed inspection method were calculated by the 3D nonlinear FEM taking account of the velocity effect. When the static magnetic field sensor was moved in the steel tube at $1 \mathrm{~m} / \mathrm{s}$, the directions of the eddy currents in the steel tube near the front and rear positions of the sensor were reversed. This is because the magnetic fields generated by the eddy currents in the steel tube were generated in the direction that cancels the magnetic fields from the sensor.

2. When there was an outer-side defect on the steel tube, the eddy current generated by the velocity effect decreased near the defect. By detecting the change in the flux density due to this eddy current as the voltage induced by the detection coil, it is possible to detect an outerside defect of a ferromagnetic steel tube using a static magnetic field.

The investigation of the optimal inspection speed and detectable defect sizes and shapes are future research subjects. 


\section{References}

Javier. Garcia. Martin, Jaime. Gomez. Gil and Ernesto. Vazquez. Sanchez: Sensors 11 (2011) 2525.

Y. Gotoh, K. Sakurai and N. Takahashi: IEEE Trans. Magn. 45 (2009) 4467.

M. Tohara and Y. Gotoh: IEEE Trans. Magn. 57 (2021).

4 G. S.Park and S. H. Park: IEEE Trans. Magn. 40 (2004) 663.

5 M. Zec, R. P. Uhlig, M. Ziolkowski, and H. Brauer: IEEE Trans. Magn. 49 (2013) 4785.

6 T. J. Rocha, H. G. Ramos, A. L. Ribeiro, and D. J.Pasadas: IEEE Trans. Magn. 65 (2016) 1182.

7 J. Wu, Y. Sun, B. Feng, and Y. Kang: IEEE Trans. Magn. 53 (2017).

8 Y. Gotoh, A. Kiya, and N. Takahashi: IEEE Trans. Magn. 46 (2010) 3145.

9 K. Muramatsu, T. Nakata, N. Takahashi, and K. Fujiwara: IEEE Trans. Magn. 28 (1992) 1186.

10 K. Muramatsu, T. Nakata, N. Takahashi, and K. Fujiwara: IEEE Trans. Magn. 32 (1996) 749.

\section{About the Authors}

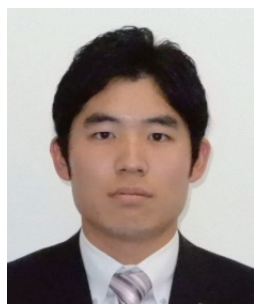

Makoto Tohara graduated from Kyushu Polytechnic College, Japan, in 2016. He received his M.E. degree from Oita University, Japan, in March 2018. Since April 2018, he has been working at TOA Non-Destructive Inspection Co., Ltd., and conducting research as part of his doctoral course at Oita University. His main research interest is non-destructive inspection using electromagnetic phenomena. (v18f1001@oita-u.ac.jp)

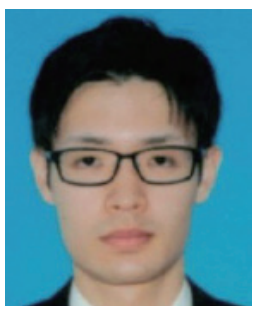

Masafumi Kuromizu graduated from Kyushu Polytechnic College, Japan, in March 2021. Since April 2021, he has been conducting research as part of his master's course at Oita University. His main research interest is the development of electromagnetic non-destructive inspection methods for steel materials.

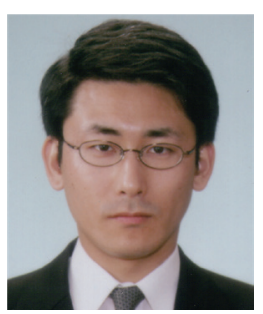

Yuji Gotoh received his B.E. and M.E. degrees from Polytechnic University, Japan, in 1996 and 1998, respectively, and his Ph.D. degree from Okayama University, Japan, in 2002. He is presently a professor in the Faculty of Science and Technology, Oita University. His main research interest is the development of electromagnetic non-destructive inspection methods for steel materials. 